\title{
Upregulation of LGMNP1 confers radiotherapy resistance in glioblastoma
}

\author{
HAO XU ${ }^{1 *}$, BINGHONG CHEN $^{2 *}$, JIN XING $^{1}$, ZILONG WEI $^{1}$, CHAOBO LIU $^{1}$, \\ YONGMING QIU ${ }^{2}$, YINGYING LIN $^{2}$ and LI REN ${ }^{1}$ \\ ${ }^{1}$ Department of Neurosurgery, Shanghai Pudong Hospital, Fudan University, Pudong, Shanghai 200120; \\ ${ }^{2}$ Department of Neurosurgery, Renji Hospital, School of Medicine, Shanghai Jiao Tong University, \\ Shanghai 200127, P.R. China
}

Received November 19, 2018; Accepted April 1, 2019

DOI: 10.3892/or.2019.7128

\begin{abstract}
Glioblastoma is a lethal brain tumor type, which is frequently resistant to radiotherapy. The aim of the present study was to explore the function of legumain pseudogene 1 (LGMNP1) on radioresistance in glioblastoma. Reverse transcription-quantitative PCR was used to detect the relative expression of LGMNP1 in glioma cell lines after radiotherapy. Ectopic expression of LGMNP1 was achieved by transfection of a lentiviral vector. A clonogenic assay was used to determine the colony formation ability following radiotherapy. A comet assay, flow cytometry and western blot analysis were applied to detect DNA damage, the apoptotic rate, and levels of apoptotic proteins, respectively. The results revealed that LGMNP1 was significantly upregulated in glioma cells after radiation. Glioma cells stably overexpressing LGMNP1 were successfully established. Overexpression of LGMNP1 in glioma cells reduced DNA damage processes and the percentage of apoptotic cells after radiotherapy. In addition, overexpression of LGMNP1 in glioblastoma multiforme cells decreased apoptotic protein expression after radiotherapy. The present results indicated that upregulation of LGMNP1 conferred radiotherapy resistance by increasing the ability of DNA damage protection and reducing the apoptotic population in glioma cells.
\end{abstract}

\section{Introduction}

Glioblastoma multiforme (GBM) is recognized as the most aggressive type of diffuse glioma of astrocytic lineage and is

Correspondence to: Dr Li Ren, Department of Neurosurgery, Shanghai Pudong Hospital, Fudan University, 2800 Gongwei Road, Pudong, Shanghai 200120, P.R. China

E-mail: slysw@sina.com

*Contributed equally

Key words: glioblastoma multiforme, legumain pseudogene 1, radioresistance, DNA damage, apoptosis equivalent to grade IV based on the World Health Organization (WHO) Classification from 2007; it is divided into isocitrate dehydrogenase (IDH) wild-type and IDH mutant-type according to molecular typing in the most recent WHO Classification (1). GBM is the most common malignancy of the central nervous system with an average annual age-adjusted incidence rate of 19/100,000 individuals, accounting for 54\% of all glioma cases (2). GBM is a refractory malignant tumor type with a median survival time of only 15 months (3). Treatment is complex, and initially consists of maximal safe surgical resection and subsequent radiation therapy with concurrent temozolomide chemotherapy, followed by 6-10 or even more cycles of maintenance temozolomide chemotherapy (4-6). Radiation therapy has a crucial role in GBM treatment, particularly for the tumor that is not totally surgically removed $(7,8)$. However, for a long time, the problem of radiotherapy resistance of GBM, which is one of the reasons why the survival of affected patients cannot be prolonged remains an obstacle for clinicians. Therefore, it is important to elucidate the mechanisms underlying radiotherapy resistance of GBM.

Legumain (LGMN), also known as asparagine endopeptidase, is a lysosomal cysteine protease originally identified in the seeds of legumes, which is also present in the human body. The gene is located on chromosome 14 and it is associated with a variety of tumor types at the stages of development, metastasis and invasion (9). A previous study by our group indicated that once tumor-associated macrophages, which highly expressed LGMN on their surface, were selectively ablated by using a doxorubicin-based prodrug activated by LGMN, tumor growth and metastasis were markedly inhibited in a murine tumor model, this implying an important role of LGMN in cancers (10). LGMN pseudogene 1 (LGMNP1) is a pseudogene of LGMN located on chromosome 13 and its expression in GBM is much higher than that in normal tissues $(11,12)$, implying that LGMNP1 has a certain association with GBM. To study the function of LGMNP1 on the radioresistance of GBM, the present study assessed whether LGMNP1 was altered after radiotherapy and whether overexpression of this gene promoted the radiotherapy resistance of GBM using in vitro experiments. In addition, the underlying mechanisms were explored. 


\section{Materials and methods}

Cell culture. The human GBM cell lines U87-MG (glioblastoma of unknown origin; cell line was authenticated by STR profiling) and T98G (purchased in 2014 from the Cell Bank of the Chinese Academy of Sciences) were cultured in Dulbecco's modified Eagle's medium (HyClone; GE Healthcare Life Sciences, Logan UT, USA) supplemented with $10 \%$ fetal bovine serum (Gibco; Thermo Fisher Scientific, Inc., Waltham, MA, USA) and maintained in a humidified atmosphere at $37^{\circ} \mathrm{C}$ with $5 \% \mathrm{CO}_{2}$.

Radiation treatment. Cells in culture were treated with an irradiator (GE 3000; GE Healthcare Life Sciences) using a ${ }^{137} \mathrm{Cs}$ source at an exact dose of 0 or $6.0 \mathrm{~Gy}$. During irradiation, the cultures were stored in the cell culture incubator $\left(5 \% \mathrm{CO}_{2}\right.$ at $37^{\circ} \mathrm{C}$ ). The cells were harvested exactly at the end of the irradiation.

Reverse transcription-quantitative PCR. Total RNA was extracted from cellular samples using TRIzol (Invitrogen; Thermo Fisher Scientific, Inc.) according to the manufacturer's instructions. UV spectrophotometry was used to determine the RNA concentration and quality. Reverse transcription of total RNA was performed using an iScript ${ }^{\mathrm{TM}}$ cDNA Synthesis kit (Bio-Rad Laboratories, Inc., Hercules, CA, USA) following the manufacturer's instructions. A 7500 Fast PCR instrument (Applied Biosystems, Thermo Fisher Scientific, Inc.) was used for quantitative PCR amplification. The primer and probe sequences were as follows: LGMNP1 forward primer, 5'-GGA CGTGGAAGATCTGACTAACC-3', reverse primer, 5'-ATG ATGTGGCTGGTATTGGTGTAT-3' and probe, 5'-VIC-CAA GCAGTGCCGCC-MGB-3'. The probe was modified with MGB at the 3'-end. GAPDH forward primer, 5'-GAAGGA CTCATGACCACAGTCCA-3', reverse primer, 5'-GCAGGG ATGATGTTCTGGAGAG-3' and probe, 5'-ROX-CGGCCA TCACGCCACAGTTTCC-3'-BHQ2. Gene alignments and primer specificity analysis were used to choose specific primers and probes. The composition of the reaction mixture contained $10 \mu \mathrm{l}$ of $2 \mathrm{X}$ TaqMan Universal Master Mix II with UNG (Applied Biosystems, Thermo Fisher Scientific, Inc.), $1 \mu \mathrm{l}(100 \mathrm{ng})$ of cDNA, $1 \mu \mathrm{l}$ of probe-primer mix and $8 \mu \mathrm{l}$ of nuclease-free water, constituting to a final volume of $20 \mu 1$. The thermocycling conditions for qPCR were as follows: $50^{\circ} \mathrm{C}$ for $2 \mathrm{~min}, 95^{\circ} \mathrm{C}$ for $10 \mathrm{~min}$ and 45 cycles of $95^{\circ} \mathrm{C}$ for $15 \mathrm{sec}$ and $60^{\circ} \mathrm{C}$ for $1 \mathrm{~min}$. Data were acquired at the end of the annealing/extension phase. Melting curve analysis was performed at the end of each run from 50 to $95^{\circ} \mathrm{C}$.

Lentiviral vector-mediated gene overexpression. The LGMNP1 overexpression sequence was constructed by Shanghai Hanyin Co., Ltd. (Shanghai, China). The recombinant lentivirus and negative control (NC) lentivirus were prepared and titered to $10^{9}$ transfection $\mathrm{U} / \mathrm{ml}$. After $48 \mathrm{~h}$, the efficiency of overexpression was confirmed via RT-qPCR. To obtain stably transfected cells (LGMNP1-OE), GBM cells were seeded in 6-well dishes at a density of $1 \times 10^{5}$ cells/well. The cells were then infected with the same virus titer on the following day with $8 \mu \mathrm{g} / \mathrm{ml}$ Polybrene. At $72 \mathrm{~h}$ post-viral infection, the culture medium was replaced with selection medium containing $4 \mu \mathrm{g} / \mathrm{ml}$ puromycin. The puromycin-resistant cells were amplified in medium containing $2 \mu \mathrm{g} / \mathrm{ml}$ puromycin for 7 days and then transferred to medium without puromycin.

Colony formation assay. The isolated cells were seeded at 300 cells/well in a 6-well tissue culture plate and grown for 14 days until macroscopic cell clones were visible. The cells were then fixed with $95 \%$ cold methanol for $15 \mathrm{~min}$ at $4^{\circ} \mathrm{C}$ and stained with $0.5 \%$ methylene blue for $2 \mathrm{~min}$ in order to determine the number of colonies by microscopy. The colony forming efficiency was calculated as the percentage of single cells that generated colonies on the 14th day. The colony formation rate was calculated as follows: Colony formation rate $=($ number of clones $/ 300) \times 100 \%$.

Comet assay. Cells were lysed by placing the slides in a Coplin jar (Thomas Scientific, Swedesboro, NJ, USA) containing $2.5 \mathrm{M} \mathrm{NaCl}, 0.1 \mathrm{M} \mathrm{Na}_{2}$ EDTA, $0.1 \mathrm{M}$ Tris and $1 \%$ Triton X-100 $(\mathrm{pH} 10)$ at $4^{\circ} \mathrm{C}$ for at least $1 \mathrm{~h}$. Subsequently, slides were immersed in electrophoresis solution $(0.3 \mathrm{M} \mathrm{NaOH}$ and $1 \mathrm{mM} \mathrm{Na} 2$ EDTA, $\mathrm{pH}>13$ ) for $30 \mathrm{~min}$. Electrophoresis was then performed at $1.3 \mathrm{~V} / \mathrm{cm}$ for $20 \mathrm{~min}$ in the same solution. Slides were washed twice in cold phosphate-buffered saline (PBS) for $10 \mathrm{~min}$ and in water for another $10 \mathrm{~min}$. Comets were fixed by immersing the slides in $70 \%$ ethanol for $15 \mathrm{~min}$ and in absolute ethanol for a further $15 \mathrm{~min}$ prior to placing them on the bench to dry overnight. Comets were stained with SYBRGold at the dilution recommended by the manufacturer in a bath at $4^{\circ} \mathrm{C}$ with agitation. After $40 \mathrm{~min}$, SYBRGold solution was removed and the slides were rinsed twice with water and left to dry at room temperature. On the day of analysis, gels were hydrated by adding a drop of water on top of each minigel, and a glass coverslip (24x60 mm) was used to cover all the minigels on the slide. The semi-automated image analysis system Comet Assay IV (Perceptive Instruments Ltd., Bury St. Edmunds, UK) was used to evaluate 100 comets/gel in the case of the $\mathrm{H}_{2} \mathrm{O}_{2}$ experiments. Percentage DNA in the tail was the parameter selected to describe each comet. The number of comets per gel (including so-called hedgehogs) was counted by direct observation.

Cell apoptosis analysis. Apoptosis was analyzed by translocation of phosphatidylserine to the cell surface using an Annexin and DAPI apoptosis detection kit (BD Biosciences, Franklin Lakes, NJ, USA). Cells were treated with 6 Gy of radiation, then collected and washed in cold PBS. Cells were resuspended in Annexin V-FITC and DAPI for $30 \mathrm{~min}$ in the dark. Cell apoptosis was analyzed on a FACSAria flow cytometer (BD Biosciences) and quantified using CellQuest software 5.1 (BD Biosciences). Fluorescence was captured with an excitation wavelength of $480 \mathrm{~nm}$.

Western blot analysis. The total proteins of cells were extracted with cell lysis buffer $(50 \mathrm{mM}$ Tris- $\mathrm{HCl} \mathrm{pH} 8.0$, $120 \mathrm{mM} \mathrm{NaCl}, 0.5 \% \mathrm{NP}-40$ and $1 \mathrm{mM}$ PMSF) and determined by BCA methods. Protein samples $(30 \mu \mathrm{g})$ were subjected to $10 \%$ SDS-PAGE and transferred to polyvinylidene difluoride membranes (EMD Millipore, Billerica, MA, USA). The membranes were incubated with blocking buffer [5\% skimmed milk in Tris-buffered saline containing Tween-20 (TBS-T)] at 

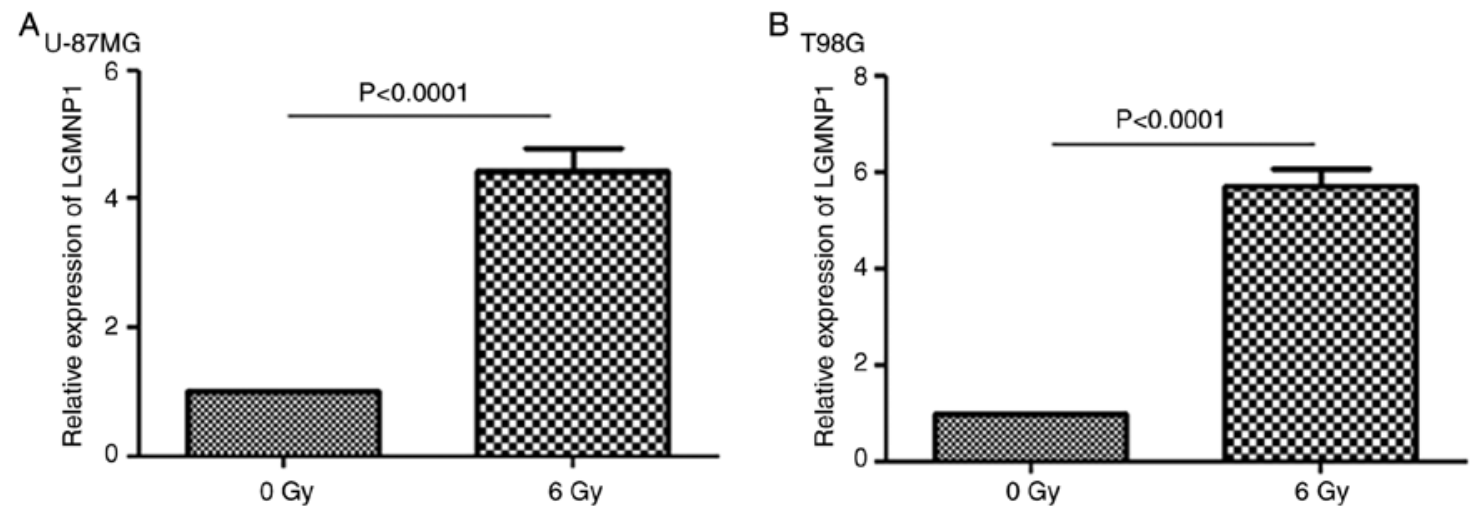

Figure 1. LGMNP1 is significantly upregulated in glioma cells after radiation. (A) Relative LGMNP1 mRNA levels in U87-MG cells treated with 6 or 0 Gy. (B) Relative LGMNP1 mRNA levels in T98G cells treated with 6 or 0 Gy. LGMNP1, legumain pseudogene 1.
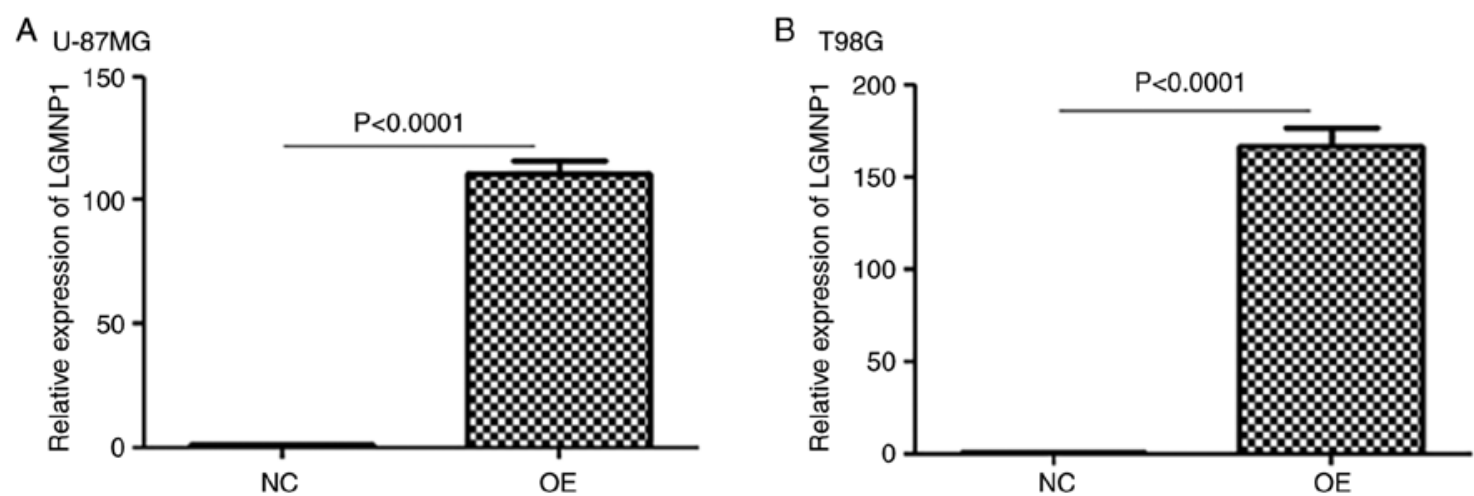

Figure 2. Overexpression of LGMNP1 in glioma cells. (A) Relative LGMNP1 mRNA levels in U87-MG cells. (B) Relative LGMNP1 mRNA levels in T98G cells. LGMNP1, legumain pseudogene 1 .

room temperature for $1 \mathrm{~h}$. The membranes were then incubated with the following antibodies at a 1:500 dilution at $4^{\circ} \mathrm{C}$ overnight: caspase-3 antibody (cat. no. 9662), caspase-7 antibody (cat. no. 12827), Bax antibody (cat. no. 14796; all from Cell Signaling Technology, Inc., Danvers, MA, USA), active caspase-3 antibody (cat. no. ab2302; Abcam, Cambridge, UK) and $\beta$-actin antibody (cat. no. 4970; Cell Signaling Technology, Inc.). The membranes were washed with TBS-T, then incubated with horseradish peroxidase-conjugated anti-rabbit (cat. no. R2655) or anti-mouse antibody (cat. no. M8270; both from Sigma-Aldrich; Merck KGaA, Darmstadt, Germany) at a 1:10,000 dilution at room temperature for $2 \mathrm{~h}$. Detection was performed using western blot detection reagents (Odyssey; LI-COR Biosciences, Lincoln, NE, USA).

Statistical analysis. Values are expressed as the mean \pm standard error of the mean. One-way analysis of variance (ANOVA) with Tukey's post hoc test was performed for comparison of multiple groups. All statistical analyses were performed using SPSS for Windows v. 17.0 (SPSS, Inc., Chicago, IL, USA). A two-tailed $\mathrm{P}<0.05$ was considered to indicate a statistically significant difference.

\section{Results}

LGMNP1 is significantly upregulated in response to radiotherapy. To determine the potential role of LGMNP1 in GBM radiotherapy resistance, RT-qPCR was employed to detect the relative expression of LGMNP1 in two glioma cell lines after exposure to a radiation dose of $6 \mathrm{~Gy}$ compared with that in the control group ( 0 Gy) (Fig. 1A and B). The result indicated that in each of the two cell lines, the relative expression of LGMNP1 in the experimental group was significantly higher than that in the control group $(\mathrm{P}<0.0001)$. It was, therefore, indicated that the expression of LGMNP1 is associated with radiotherapy.

Upregulation of LGMNP1 enhances the colony formation ability after radiotherapy. To verify the function of LGMNP1 in radiotherapy resistance of GBM, U87-MG and T98G cells with stable overexpression of LGMNP1 were established using the lentiviral vector-mediated method. As is presented in Fig. 2A and B, LGMNP1 was effectively upregulated in LGMNP1-OE cells compared with that in the NC cells. The clonogenic assay indicated that the colony formation ability of LGMNP1-OE cells after radiotherapy was greater than that of the NC cells (Fig. 3). These results suggested that overexpression of LGMNP1 contributes to radiation resistance.

Overexpression of LGMNPI confers radioresistance by enhancing the ability of DNA damage protection and reduction of apoptosis. One of the mechanisms by which radiotherapy kills tumor cells is the induction of DNA double-strand breaks. In order to determine whether overexpression of 
A

T98G

T98g-NC

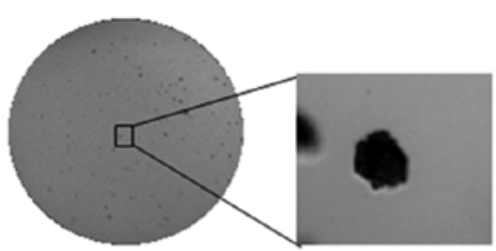

T98g-OE

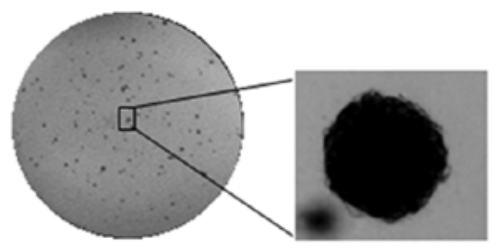

C

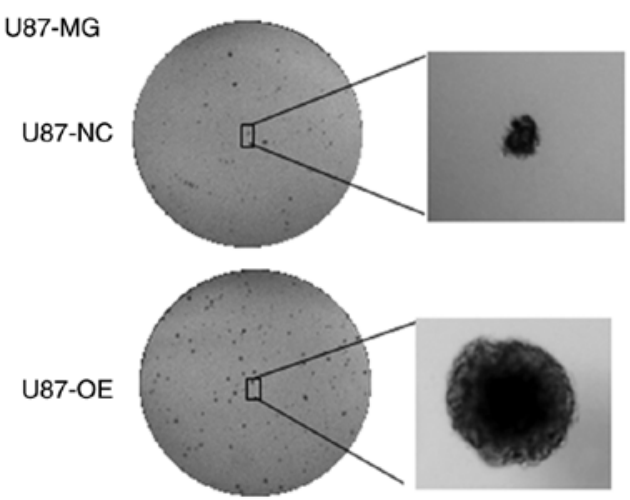

$\mathrm{B}$

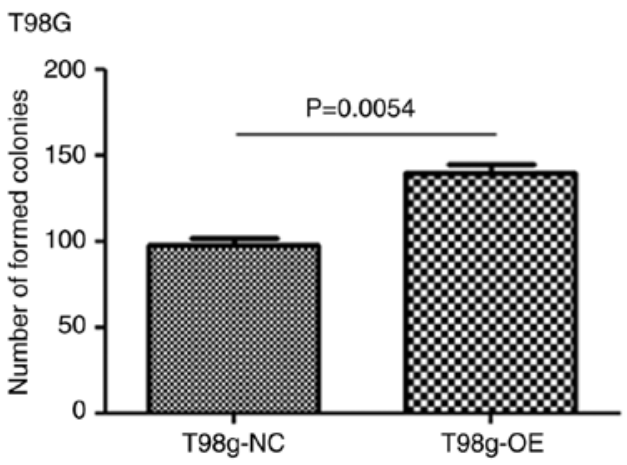

D

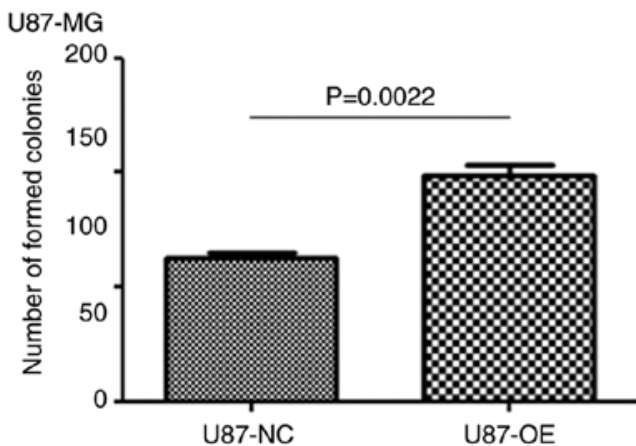

Figure 3. Overexpression of LGMNP1 in glioma cells increases the colony formation ability after radiotherapy. (A and B) Size and number of colonies formed by LGMNP1 overexpressing or native control T98G cells treated with $6 \mathrm{~Gy}$. (C and D) Size and number of colonies formed by LGMNP1 overexpressing or native control U87-MG cells treated with 6 Gy. LGMNP1, legumain pseudogene 1.

LGMNP1 improves the capacity for DNA damage protection after exposure to radiation damage, the cell lines were subjected to the comet assay. The results indicated that the percentage of tail DNA was higher in the cells exposed to 6 Gy of radiation compared with that of cells that received 0 Gy (Fig. 4). Furthermore, the percentage of tail DNA in the LGMNP1-OE group was less than that in the NC group, in the T98G cell line (Fig. 4A and B) and also in the U87-MG cell line (Fig. 4C and D). These results suggested that overexpression of LGMNP1 improves the ability of glioma cells to perform DNA damage protection. To detect apoptosis in glioma cell lines, flow cytometric analysis was employed, demonstrating a lower ratio of apoptosis in LGMNP1-OE vs. NC glioma cells after radiotherapy (Fig. 5). For further verification, the levels of apoptotic proteins were assessed by western blot analysis. The results revealed that the levels of apoptotic proteins, including caspase-3, active caspase-3, caspase-7 and Bax in LGMNP1-OE glioma cells treated with radiotherapy were decreased compared with those in the $\mathrm{NC}$ group, and almost the same results were obtained with the two cell lines (Fig. 6). Overall, these results indicated that overexpression of LGMNP1 in GBM cell lines enhances the capacity for DNA damage protection and reduces apoptosis following radiation-induced damage.

\section{Discussion}

With the development of surgical techniques and the routine use of radiotherapy and chemotherapy, the two-year survival of
GBM patients has increased from 7\% among cases diagnosed between 1993 and 1995 to $17 \%$ in cases diagnosed between 2005 and 2007 (3). In recent years, further treatments have been implemented for the treatment of GBM, among which immunotherapy, chimeric antigen receptor (CAR) T-cell therapy, tumor vaccines, viral therapy and tumor-treating fields (TTF) therapy have provided certain benefits. A patient with recurrent multifocal glioblastoma receiving CAR-engineered $T$ cells targeting the tumor-associated antigen interleukin-13 receptor $\alpha 2$ exhibited regression of all intracranial and spinal tumors (13). Tetanus toxoid pre-conditioning may improve the migration of the dendritic cell vaccine and suppress tumor growth in mice and glioblastoma patients (14). Oncolytic viruses are the most extensively studied type of virus in glioma treatment and have been developed for brain cancer treatment. They were demonstrated to be safe and such therapies may also direct long-lasting immune responses toward the tumor while reducing early antiviral reactions (15). TTF therapy has been evaluated in randomized phase 3 trials in GBM and was demonstrated to prolong progression-free survival and overall survival when administered together with standard maintenance temozolomide chemotherapy in patients with newly diagnosed GBM $(16,17)$. However, this is far from enough, all of the aforementioned methods are only effective in a limited number of patients. Therefore, traditional radiotherapy still remains crucial for the treatment of GBM. For newly diagnosed GBM, accurate, timely and efficient radiotherapy is required and for recurrent GBM, radiation is also recommended if the cancer is still susceptible (18-20). The resistance of GBM to 
A
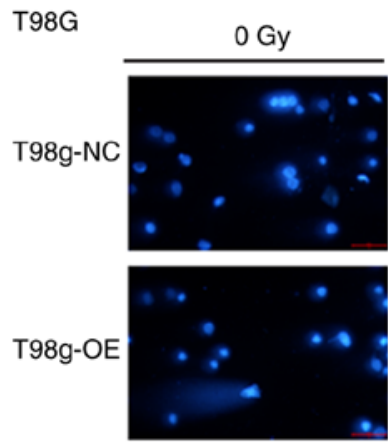

C U87-MG

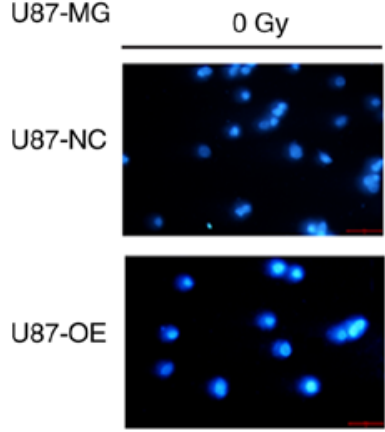

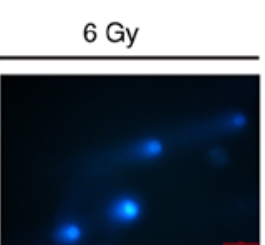

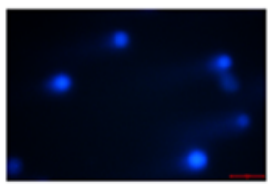

6 Gy
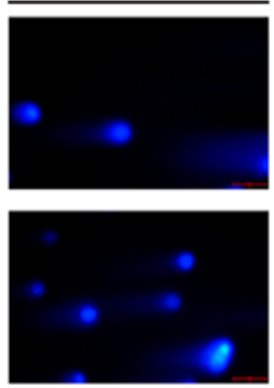

B

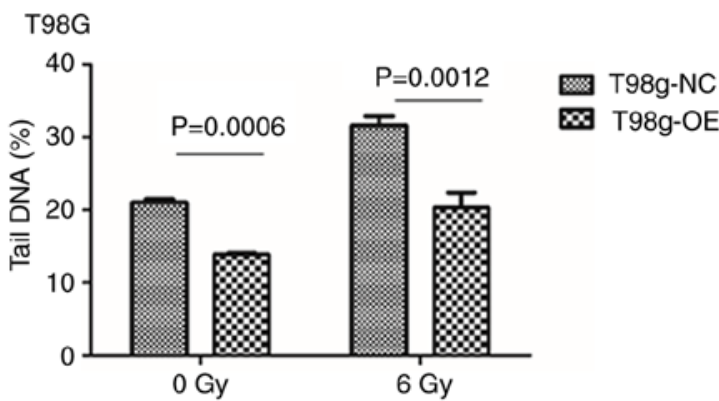

D

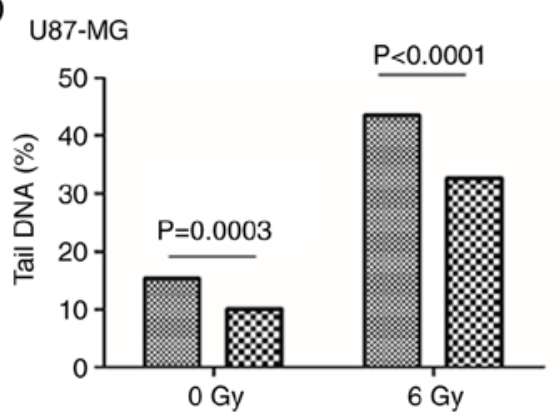

Figure 4. Overexpression of LGMNP1 in glioma cells enhances DNA damage protection processes after radiotherapy. (A) Fluorescent comet tails of LGMNP1-OE or NC T98G cells treated with 6 or 0 Gy. (B) Tail DNA (\%) of LGMNP1-OE or NC T98G cells treated with 6 or 0 Gy. (C) Fluorescent comet tails of LGMNP1-OE or NC U87-MG cells treated with 6 or 0 Gy. (D) Tail DNA (\%) of LGMNP1-OE or NC U87-MG cells treated with 6 or 0 Gy. LGMNP1-OE, legumain pseudogene 1 overexpression; $\mathrm{NC}$, negative control.
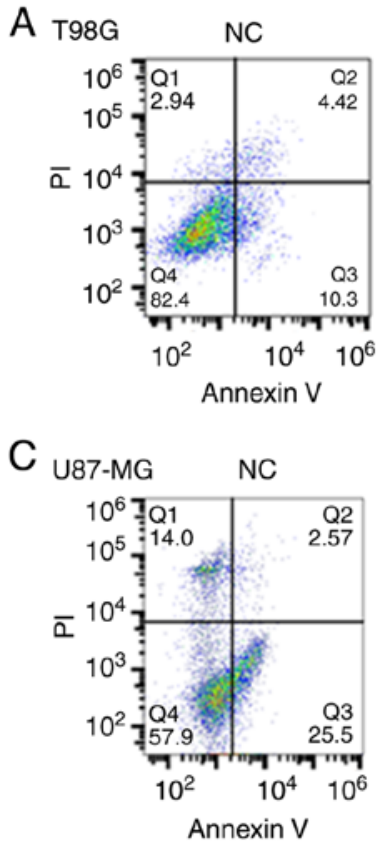
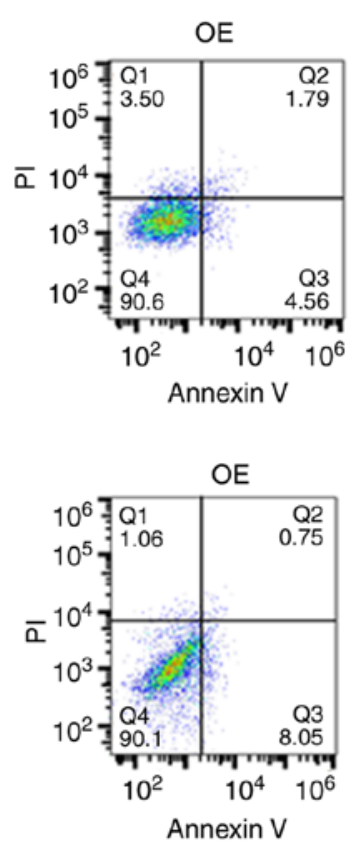
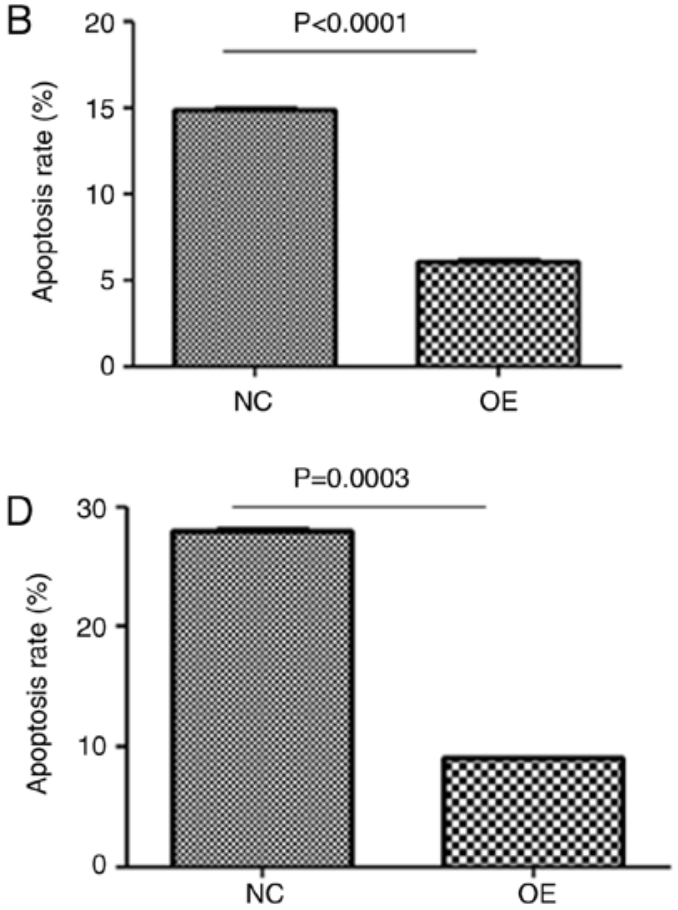

Figure 5. Overexpression of LGMNP1 in glioblastoma multiforme cells reduces the apoptotic cell population after radiotherapy. (A and B) Apoptosis rate of LGMNP1-OE or NC T98G cells after radiotherapy. (C and D) Apoptosis rate of LGMNP1-OE or NC U87-MG cells after radiotherapy. LGMNP1, legumain pseudogene 1.

radiation therapy is a common problem, and the underlying mechanisms remain to be fully elucidated. For tumor cells, abnormal activation of the DNA damage repair pathway is not only the root cause of tumorigenesis, but also an important mechanism for its resistance to radiotherapy. p53, as a widely known tumor suppressor gene interacts with Rad51 promoter and Rad51 protein to downregulate Rad51, thereby inhibiting homologous recombination and DNA repair (21). After 
A

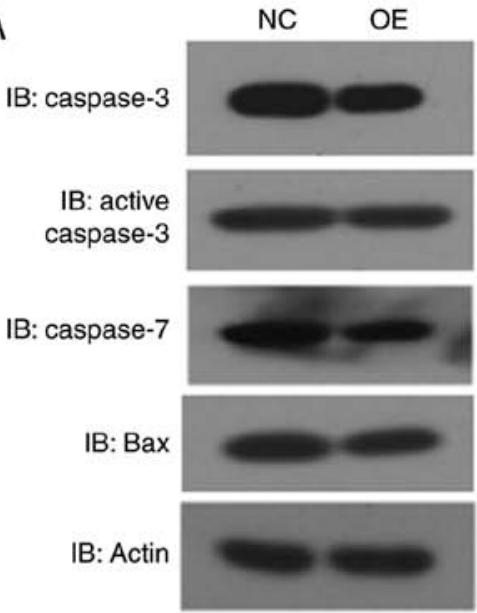

B
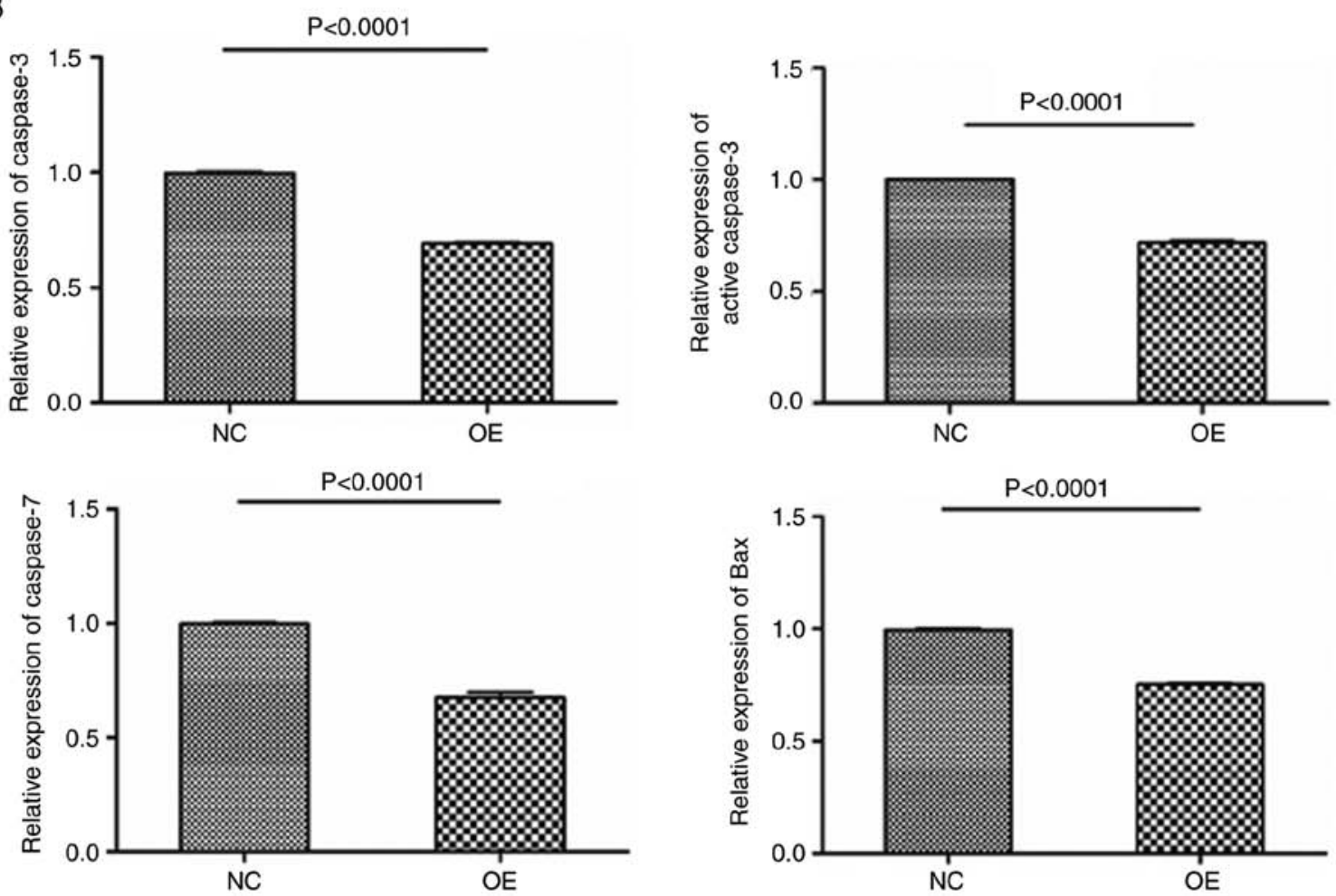

Figure 6. Overexpression of LGMNP1 in glioblastoma multiforme cells decreases apoptotic proteins after radiotherapy. (A) Protein bands of caspase-3, active caspase-3, caspase-7, Bax and $\beta$-actin from LGMNP1-OE or NC T98G cells after radiotherapy. (B) Relative expression of caspase-3, active caspase-3, caspase-7 and Bax in LGMNP1-OE or NC T98G cells after radiotherapy.

radiation, an accelerated senescence response was observed in p53 wild-type GBM cells (22). In addition, it is known that glioma stem cells expressing CD133 as a biomarker contribute to radioresistance of glioma through preferential activation of the DNA damage checkpoint response and an increase in DNA repair capacity (23). Beyond that, vascular endothelial growth factor (VEGF) has been thoroughly studied, since it is one of the angiogenic growth factors that is highly expressed in GBM. A tumor blood flow study indicated that the blood volume increased following radiotherapy, which may be associated with the expression of VEGF (24). Furthermore, VEGF binds to the vascular endothelial growth factor receptor 1 and activates a signal transduction cascade, neutralizing antibodies to the receptor inhibited angiogenesis and enhanced the radiation-induced response, and a greater additive effect was achieved in GBM when VEGF blocking antibody was combined with radiation $(25,26)$. Advanced research on the epidermal growth factor receptor (EGFR) using in vitro and in vivo experiments indicated that high levels of EGFR or EGFRvIII were able to facilitate DNA double-strand break repair, which was mediated by the AKT pathway (26-28). The occurrence of tumor radiotherapy resistance is thought not to be a single signaling process. Studies have revealed that blockade of transforming growth factor $\beta$ signaling enhanced the response of glioblastoma patients to radiation therapy and prolonged their survival (29). Furthermore, combined inhibition of poly(ADP-ribose) polymerase and heat shock protein 90 enhanced the radiosensitivity of human glioma cells (30). The mechanisms require to be elucidated in further studies. 

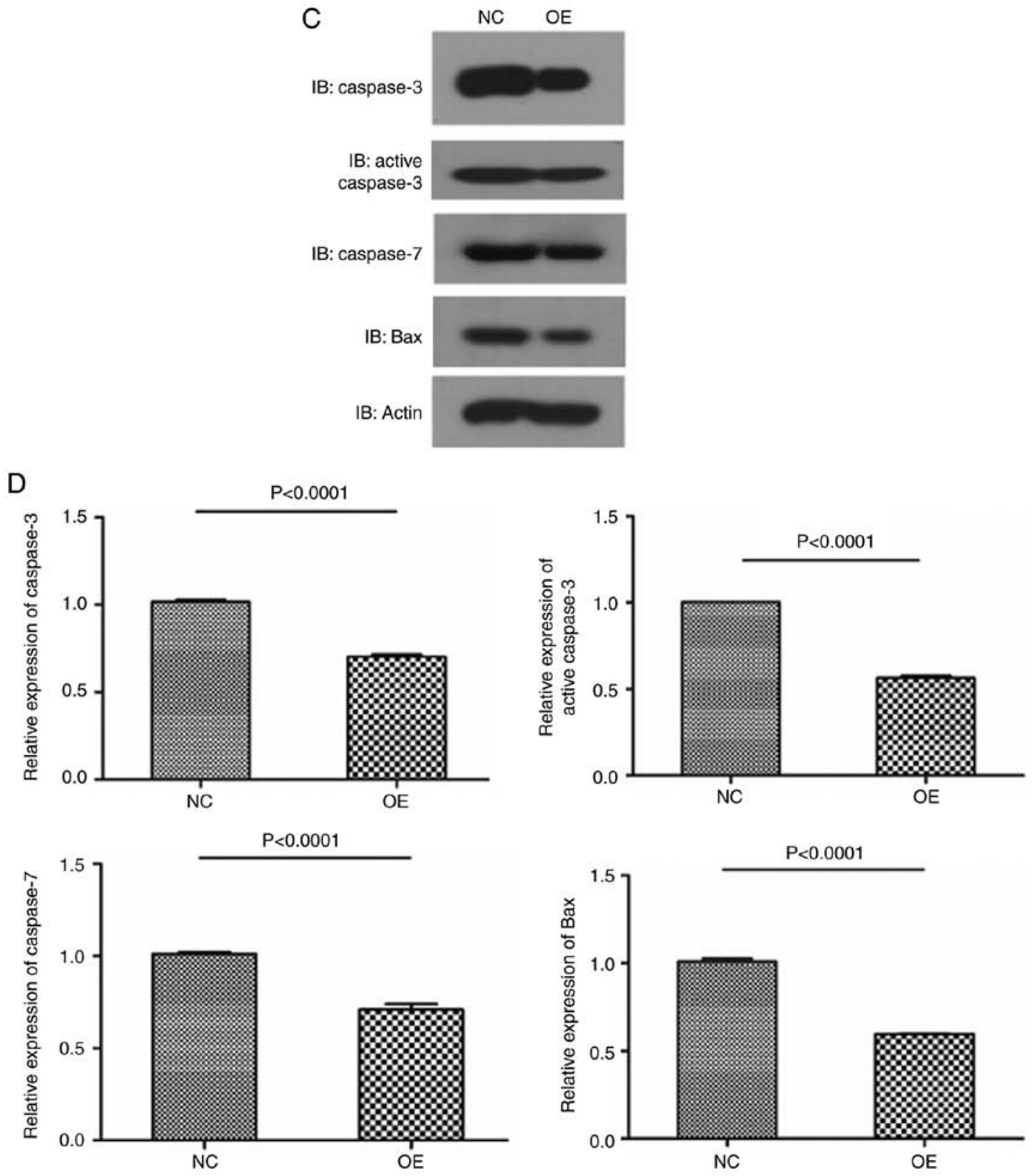

Figure 6. Continued. (C) Protein bands of caspase-3, active caspase-3, caspase-7, Bax and $\beta$-actin from LGMNP1-OE or NC U87-MG cells after radiotherapy. (D) Relative expression of caspase-3, active caspase-3, caspase-7 and Bax in LGMNP1-OE or NC U87-MG cells after radiotherapy. LGMNP1-OE, legumain pseudogene 1 overexpression; $\mathrm{NC}$, negative control.

LGMN is known for its cysteine endopeptidase activity in lysosomes, where it contributes to antigen processing for class II major histocompatibility complex presentation. However, it also occurs extracellularly and even translocates to the cytosol and the nucleus. LGMN has also been reported to be associated with the development of a variety of tumor types, including breast cancer, gastric carcinoma, ovarian and colorectal cancer, and it may even serve as a biomarker for such tumors (31-34). Furthermore, LGMN was indicated to promote the proliferation and invasiveness of prostate cancer cells via the PI3K/AKT signaling pathway (35). In addition, knockdown of LGMN was reported to suppress cervical cancer cell migration and invasion (36). Pseudogenes are not truly non-functional genes, although they do not encode proteins, but non-coding RNAs formed by transcription have certain functions. It has been indicated that abnormal expression of pseudogenes may have vital roles in tumors (37). For instance, phosphatase and tensin homolog (PTEN) is under the regulatory control of PTEN pseudogene-expressed non-coding RNA-PTENpg1, which encodes antisense RNA (asRNA) that regulates PTEN transcription by balancing the two PTENpg1 asRNA isoforms, $\alpha$ and $\beta$ (38). It is possible that LGMNP1 regulates LGMN expression in a similar manner to enhance the DNA repair capacity and produce radiotherapy resistance. According to the results of the present study, LGMNP1 was upregulated once the glioma cells were exposed to radiotherapy, and when LGMNP1 was ectopically overexpressed, the glioma cells were more resistant to radiotherapy. The comet assay and the apoptosis detection assays indicated less amount of DNA double-strand 
breaks and the amounts of apoptotic cells and proteins were decreased. In summary, upregulation of LGMNP1 was indicated to enhance the radioresistance of glioma and targeting LGMNP1 may be a novel strategy to increase sensitivity to radiotherapy, however the mechanism still requires further exploration.

In conclusion, LGMNP1 was upregulated in GBM cell lines after administration of ionizing radiation. LGMNP1 confered radiotherapy resistance by increasing the capacity for DNA damage protection and reducing apoptosis in glioma cells in vitro. The therapy targeting LGMNP1 may be a promising method to reverse radioresistance of GBM.

\section{Acknowledgements}

Not applicable.

\section{Funding}

The present study was funded by the National Natural Science Foundation of China (grant nos. 81402042 and 81772654), the Shanghai Pudong New Area Science and Technology Development Fund (grant no. PKJ2016-Y45), the Shanghai Pudong New Area Health and Family Planning Commission (grant nos. PWZzk2017-16 and PWRL2017-03) and the Shanghai Science and Technology Committee (grant no. 16140902900).

\section{Availability of data and materials}

The datasets used during the present study are available from the corresponding author upon reasonable request.

\section{Authors' contributions}

LR and YL designed and supervised the research, YQ was also involved in the conception of the study. $\mathrm{HX}$ and $\mathrm{BC}$ took part in the fund raising, experimental design, data acquisition, manuscript writing, performed the majority of the experiments and drafted the manuscript. ZW and JX helped with the design and performance of the experiments of western blotting and lentiviral vector-mediated gene overexpression. CL helped with the colony formation and comet assays. YL and YQ contributed to the revising of the manuscript. All authors were involved in the conception of the study, read and approved the manuscript and agree to be accountable for all aspects of the research in ensuring that the accuracy or integrity of any part of the work are appropriately investigated and resolved.

\section{Ethics approval and consent to participate}

Not applicable

\section{Patient consent for publication}

Not applicable

\section{Competing interests}

The authors declare that they have no competing interests.

\section{References}

1. Louis DN, Perry A, Reifenberger G, von Deimling A, Figarella-Branger D, Cavenee WK, Ohgaki H, Wiestler OD, Kleihues P and Ellison DW: The 2016 World Health Organization classification of tumors of the central nervous system: A summary. Acta neuropathol 131: 803-820, 2016.

2. Ostrom QT, Gittleman H, Farah P, Ondracek A, Chen Y, Wolinsky Y, Stroup NE, Kruchko C and Barnholtz-Sloan JS: CBTRUS statistical report: Primary brain and central nervous system tumors diagnosed in the United States in 2006-2010. Neuro Oncol 15 (Suppl 2): ii1-ii56, 2013.

3. Darefsky AS, King JT Jr and Dubrow R: Adult glioblastoma multiforme survival in the temozolomide era: A population-based analysis of Surveillance, Epidemiology, and End Results registries. Cancer 118: 2163-2172, 2012.

4. Thakkar JP, Dolecek TA, Horbinski C, Ostrom QT, Lightner DD, Barnholtz-Sloan JS and Villano JL: Epidemiologic and molecular prognostic review of glioblastoma. Cancer Epidemiol Biomarkers Prev 23: 1985-1996, 2014.

5. Stupp R, Dietrich PY, Ostermann Kraljevic S, Pica A, Maillard I, Maeder P, Meuli R, Janzer R, Pizzolato G, Miralbell R, et al: Promising survival for patients with newly diagnosed glioblastoma multiforme treated with concomitant radiation plus temozolomide followed by adjuvant temozolomide. J Clin Oncol 20: 1375-1382, 2002.

6. Stupp R, Mason WP, van den Bent MJ, Weller M, Fisher B, Taphoorn MJ, Belanger K, Brandes AA, Marosi C, Bogdahn U, et al: Radiotherapy plus concomitant and adjuvant temozolomide for glioblastoma. N Engl J Med 352: 987-996, 2005.

7. Wind JJ, Young R, Saini A and Sherman JH: The role of adjuvant radiation therapy in the management of high-grade gliomas. Neurosurg Clin N Am 23: 247-258, viii, 2012.

8. Caruso C, Carcaterra M and Donato V: Role of radiotherapy for high grade gliomas management. J Neurosurg Sci 57: 163-169, 2013.

9. Zhen Y, Chunlei G, Wenzhi S, Shuangtao Z, Na L, Rongrong W, Xiaohe L, Haiying N, Dehong L, Shan J, et al: Clinicopathologic significance of legumain overexpression in cancer: A systematic review and meta-analysis. Sci Rep 5: 16599, 2015.

10. Lin Y, Wei C, Liu Y, Qiu Y, Liu C and Guo F: Selective ablation of tumor-associated macrophages suppresses metastasis and angiogenesis. Cancer Sci 104: 1217-1225, 2013.

11. Cancer Genome Atlas Research Network; Weinstein JN, Collisson EA, Mills GB, Shaw KR, Ozenberger BA, Ellrott K, Shmulevich I, Sander C, Stuart JM, et al: The Cancer Genome Atlas Pan-Cancer analysis project. Nat Genet 45: 1113-1120, 2013.

12. Zheng LL, Zhou KR, Liu S, Zhang DY, Wang ZL, Chen ZR, Yang JH and Qu LH: dreamBase: DNA modification, RNA regulation and protein binding of expressed pseudogenes in human health and disease. Nucleic Acids Res 46: D85-D91, 2018.

13. Brown CE, Alizadeh D, Starr R, Weng L, Wagner JR, Naranjo A, Ostberg JR, Blanchard MS, Kilpatrick J, Simpson J, et al: Regression of glioblastoma after chimeric antigen receptor t-cell therapy. N Engl J Med 375: 2561-2569, 2016.

14. Mitchell DA, Batich KA, Gunn MD, Huang MN, Sanchez-Perez L, Nair SK, Congdon KL, Reap EA, Archer GE, Desjardins A, et al: Tetanus toxoid and CCL3 improve dendritic cell vaccines in mice and glioblastoma patients. Nature 519: 366-369, 2015.

15. Kaufmann JK and Chiocca EA: Glioma virus therapies between bench and bedside. Neuro Oncol 16: 334-351, 2014.

16. Hottinger AF, Pacheco P and Stupp R: Tumor treating fields: a novel treatment modality and its use in brain tumors. Neuro Oncol 18: 1338-1349, 2016.

17. Stupp R, Taillibert S, Kanner AA, Kesari S, Steinberg DM, Toms SA, Taylor LP, Lieberman F, Silvani A, Fink KL, et al: Maintenance therapy with tumor-treating fields plus temozolomide vs temozolomide alone for glioblastoma: A randomized clinical trial. JAMA 314: 2535-2543, 2015.

18. Ryu S, Buatti JM, Morris A, Kalkanis SN, Ryken TC and Olson JJ; AANS/CNS Joint Guidelines Committee: The role of radiotherapy in the management of progressive glioblastoma: A systematic review and evidence-based clinical practice guideline. J Neurooncol 118: 489-499, 2014.

19. Lutz ST, Jones J and Chow E: Role of Radiation Therapy in Palliative Care of the Patient With Cancer. J Clin Oncol 32: 2913-2919, 2014.

20. Gallego O: Nonsurgical treatment of recurrent glioblastoma. Curr Oncol 22: E273-E281, 2015. 
21. Arias-Lopez C, Lazaro-Trueba I, Kerr P, Lord CJ, Dexter T, Iravani M, Ashworth A and Silva A: p53 modulates homologous recombination by transcriptional regulation of the RAD51 gene. EMBO Rep 7: 219-224, 2006.

22. Quick QA and Gewirtz DA: An accelerated senescence response to radiation in wild-type p53 glioblastoma multiforme cells J Neurosurg 105: 111-118, 2006

23. Bao S, Wu Q, McLendon RE, Hao Y, Shi Q, Hjelmeland AB Dewhirst MW, Bigner DD and Rich JN: Glioma stem cells promote radioresistance by preferential activation of the DNA damage response. Nature 444: 756-760, 2006.

24. Gorski DH, Beckett MA, Jaskowiak NT, Calvin DP, Mauceri HJ, Salloum RM, Seetharam S, Koons A, Hari DM, Kufe DW, et al: Blockade of the vascular endothelial growth factor stress response increases the antitumor effects of ionizing radiation. Cancer Res 59: 3374-3378, 1999.

25. Geng L, Donnelly E, McMahon G, Lin PC, Sierra-Rivera E Oshinka $\mathrm{H}$ and Hallahan DE: Inhibition of vascular endothelia growth factor receptor signaling leads to reversal of tumor resistance to radiotherapy. Cancer Res 61: 2413-2419, 2001.

26. Hatanpaa KJ, Burma S, Zhao D and Habib AA: Epidermal growth factor receptor in glioma: Signal transduction, neuropathology, imaging, and radioresistance. Neoplasia 12: 675-684, 2010.

27. Mukherjee B, McEllin B, Camacho CV, Tomimatsu N, Sirasanagandala S, Nannepaga S, Hatanpaa KJ, Mickey B Madden C, Maher E, et al: EGFRvIII and DNA double-strand break repair: A molecular mechanism for radioresistance in glioblastoma. Cancer Res 69: 4252-4259, 2009.

28. Liccardi G, Hartley JA and Hochhauser D: Importance of EGFR/ERCC1 interaction following radiation-induced DNA damage. Clin Cancer Res 20: 3496-3506, 2014.

29. Zhang M, Kleber S, Roehrich M, Timke C, Han N, Tuettenberg J, Martin-Villalba A, Debus J, Peschke P, Wirkner U, et al: Blockade of TGF- $\beta$ signaling by the TGF $\beta$ R-I kinase inhibitor LY2109761 enhances radiation response and prolongs survival in glioblastoma. Cancer Res 71: 7155-7167, 2011.
30. Dungey FA, Caldecott KW and Chalmers AJ: Enhanced radiosensitization of human glioma cells by combining inhibition of poly(ADP-ribose) polymerase with inhibition of heat shock protein 90. Mol Cancer Ther 8: 2243-2254, 2009.

31. Wu M, Shao GR, Zhang FX, Wu WX, Xu P and Ruan ZM: Legumain protein as a potential predictive biomarker for Asian patients with breast carcinoma. Asian Pac J Cancer Prev 15: 10773-10777, 2014

32. Haugen MH, Boye K, Nesland JM, Pettersen SJ, Egeland EV, Tamhane T, Brix K, Maelandsmo GM and Flatmark K: High expression of the cysteine proteinase legumain in colorectal cancer-implications for therapeutic targeting. Eur J Cancer 51: 9-17, 2015.

33. Guo P, Zhu Z, Sun Z, Wang Z, Zheng X and Xu H: Expression of legumain correlates with prognosis and metastasis in gastric carcinoma. PLoS One 8: e73090, 2013.

34. Zhu Q, Tang M and Wang X: The expression of asparaginyl endopeptidase promotes growth potential in epithelial ovarian cancer. Cancer Biol Ther 18: 222-228, 2017.

35. Zhu W, Shao Y, Yang M, Jia M and Peng Y: Asparaginyl endopeptidase promotes proliferation and invasiveness of prostate cancer cells via PI3K/AKT signaling pathway. Gene 594: 176-182, 2016.

36. Meng F and Liu W: Knockdown of legumain suppresses cervical cancer cell migration and invasion. Oncol Res 23: 7-12, 2016.

37. Kalyana-Sundaram S, Kumar-Sinha C, Shankar S, Robinson DR, Wu YM, Cao X, Asangani IA, Kothari V, Prensner JR, Lonigro RJ, et al: Expressed pseudogenes in the transcriptional landscape of human cancers. Cell 149: 1622-1634, 2012.

38. Johnsson P, Ackley A, Vidarsdottir L, Lui WO, Corcoran M, Grandér D and Morris KV: A pseudogene long-noncoding-RNA network regulates PTEN transcription and translation in human cells. Nat Struct Mol Biol 20: 440-446, 2013. 